\title{
The magnetocaloric effect in soft magnetic amorphous alloys
}

\author{
V. Franco, a) J. S. Blázquez, M. Millán, J. M. Borrego, C. F. Conde, and A. Conde \\ Departmento de Física de la Materia Condensada, ICMSE-CSIC, Universidad de Sevilla, P.O. Box 1065, \\ 41080 Sevilla, Spain
}

(Presented on 8 January 2007; received 31 October 2006; accepted 17 November 2006; published online 23 March 2007)

\begin{abstract}
The influence of different compositional modifications on the magnetic entropy change and refrigerant capacity of Finemet, Nanoperm, HiTperm, and bulk amorphous alloys is presented. For all the studied alloys, the field dependence of the magnetic entropy change exhibits a quadratic dependence in the paramagnetic regime, a linear dependence in the ferromagnetic temperature range, and a potential law with a field exponent $\sim 0.75$ at the Curie temperature. This exponent can be explained using the critical exponents of the Curie transition. It is shown that for alloys of similar compositional series, the magnetic entropy change follows a master curve behavior. (C) 2007 American Institute of Physics. [DOI: 10.1063/1.2709409]
\end{abstract}

Magnetic refrigeration is a field of current interest due to the enhanced performance and reduced environmental impact of these systems when compared with those based on the gas compression-expansion cycle. ${ }^{1-3}$ Although current prototypes are based on rare-earth-based materials, ${ }^{4}$ soft magnetic amorphous alloys have been recently proposed as low cost candidates for high temperature magnetic refrigeration. $^{5-13}$

Albeit the maximum magnetic entropy change, $\left|\Delta S_{M}^{\mathrm{pk}}\right|$, for these alloys is modest when compared to that of rareearth-based materials, ${ }^{14,15}$ the remarkable difference in material costs is an incentive for studying their suitability as magnetic refrigerants. Besides their reduced magnetic hysteresis (virtually negligible), higher electrical resistivity (which would decrease eddy current losses), tunable Curie temperature and, in the case of bulk amorphous alloys, outstanding mechanical properties are beneficial characteristics for a successful application of the material. The field dependence of the magnetic entropy change $\Delta S_{M}$ in materials with a second order phase transition has also been recently studied, demonstrating that a master curve behavior is fulfilled for $\Delta S_{M}$ curves measured up to different maximum fields. ${ }^{16}$ The aim of this work is twofold: to present a comparison of the relevant characteristics of the magnetocaloric response of selected Finemet, Nanoperm, HiTperm, and bulk amorphous alloys (BAAs) prepared in ribbon shape and to demonstrate that $\Delta S_{M}(T)$ curves of different alloys from the same compositional series can also collapse in a master curve.

Amorphous alloys from different compositional series have been prepared in ribbon shape $(\sim 25 \mu \mathrm{m}$ thick) by melt spinning: $\mathrm{Fe}_{x} \mathrm{Co}_{y} \mathrm{~B}_{z} \mathrm{C}_{u} \mathrm{Si}_{3} \mathrm{Al}_{5} \mathrm{Ga}_{2} \mathrm{P}_{10} \quad(x, y, z, u=70,0,5,5$; $56,14,6,4 ; 43,26,8,3 ; 29,40,9,2 ; 17,52,10,1 ; 5,63,12,0 ;)$, denoted as CoBAA; ${ }^{11} \mathrm{Fe}_{65.5} \mathrm{Cr}_{4-x} \mathrm{Mo}_{4-y} \mathrm{Cu}_{x+y} \mathrm{Ga}_{4} \mathrm{P}_{12} \mathrm{C}_{5} \mathrm{~B}_{5.5}(x$, $y=0 ; \quad 0.5 ; \quad 1, \quad x+y \leqslant 1), \quad$ denoted as CrMoBAA; ${ }^{12}$ $\mathrm{Fe}_{83-x} \mathrm{Co}_{x} \mathrm{Zr}_{6} \mathrm{~B}_{10} \mathrm{Cu}_{1}(x=0,5)$, denoted as CoNanoperm; ${ }^{10}$ $\mathrm{Fe}_{91-x} \mathrm{Mo}_{8} \mathrm{Cu}_{1} \mathrm{~B}_{x}(x=15,17,20)$, denoted as BNanoperm; ${ }^{17}$ $\mathrm{Fe}_{60-x} \mathrm{Mn}_{x} \mathrm{Co}_{18} \mathrm{Nb}_{6} \mathrm{~B}_{16} \quad(x=0, \quad 2,4)$, denoted as

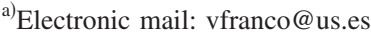

MnHiTperm; ${ }^{18}$ and $\mathrm{Fe}_{68.5} \mathrm{Mo}_{5} \mathrm{Si}_{13.5} \mathrm{~B}_{9} \mathrm{Cu}_{1} \mathrm{Nb}_{3}$, denoted as MoFinemet. ${ }^{8}$ Their amorphous character was checked by $\mathrm{X}$-ray diffraction. The specific heat $\left(c_{p}\right)$ of the CoNanoperm series has been measured in a Perkin-Elmer DSC-7 differential scanning calorimeter (DSC) using $20 \mathrm{~K} / \mathrm{min}$ scans. Samples were placed in $\mathrm{Al}$ pans and the $c_{p}$ absolute values were obtained after baseline subtraction (recorded signal of an empty $\mathrm{Al}$ pan) and the calibration of the DSC signal using as a standard a sapphire sample in an Al pan. The field dependence of magnetization was measured in a Lakeshore 7407 vibrating sample magnetometer using a maximum applied field $H=1.5 \mathrm{~T}$ with field steps of $5 \mathrm{mT}$, for constant temperatures in the range of 300-720 K with increments of $10 \mathrm{~K}$. Prior to the measurements, the stress of the samples was relaxed by preannealing.

The magnetic entropy change due to the application of a magnetic field $H$ has been evaluated from the processing of the temperature and field dependent magnetization curves using a numerical approximation to the equation:

$$
\Delta S_{M}=\int_{0}^{H}\left(\frac{\partial M}{\partial T}\right)_{H} d H,
$$

where the partial derivative is replaced by finite differences and the integration is performed numerically.

In order to compare the performance of different materials, either $\left|\Delta S_{M}^{\mathrm{pk}}\right|$ or the refrigerant capacity (RC) is used. The refrigeration at low temperatures requires a narrow temperature span of the refrigeration cycle, making $\left|\Delta S_{M}^{\mathrm{pk}}\right|$ the parameter of choice for comparing low temperature materials, while high temperature refrigeration implies a wider temperature range and, consequently, $\mathrm{RC}$ is employed for comparison. According to Wood and Potter, ${ }^{19}$ the RC of a reversible refrigeration cycle operating between $T_{h}$ and $T_{c}$ (the temperatures of the hot and cold reservoirs, respectively) is defined as $\mathrm{RC}=\left|\Delta S_{M}\right|\left(T_{h}-T_{c}\right)$, where $\left|\Delta S_{M}\right|$ is the magnetic entropy change at the hot and cold ends of the cycle. Moreover, hysteresis losses can be taken into account when evaluating the refrigerant material by subtracting them from the computed $\mathrm{RC},{ }^{15}$ making the comparison between materi- 


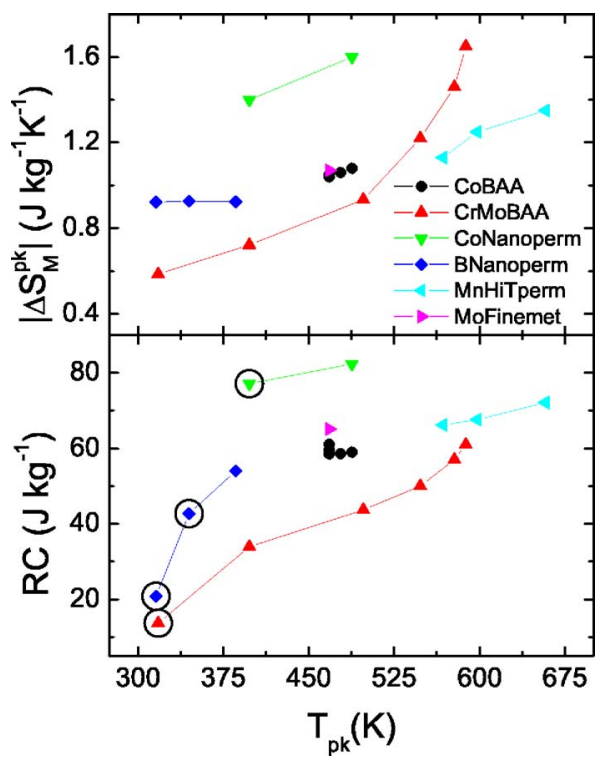

FIG. 1. (Color online) Relationship between the peak temperature of the magnetic entropy change and the magnitude of the peak (upper) and the refrigerant capacity (lower) for different alloy series for a maximum applied field of $1.5 \mathrm{~T}$. Lines are a guide to the eyes.

als with different coercivities more straightforward. The optimal refrigeration cycle is that which maximizes the RC.

Figure 1 shows the dependence of $\left|\Delta S_{M}^{\mathrm{pk}}\right|$ and RC with the peak temperature $T_{\mathrm{pk}}$ of the $\Delta S_{M}$ curves. Circles around some of the RC points indicate that for that particular alloy $\mathrm{RC}$ does not reach a maximum when measured in the available temperature range due to the proximity between $T_{\mathrm{pk}}$ and room temperature. The optimal materials would be those which maximize both parameters and present a peak temperature close to the desired operational range. For the design of composite materials with a broad operational range, $\left|\Delta S_{M}^{\mathrm{pk}}\right|\left(T_{\mathrm{pk}}\right)$ should remain as flat as possible. For most of the studied alloys, compositional changes that produce a reduction in $T_{\mathrm{pk}}$ also cause a reduction in both $\left|\Delta S_{M}^{\mathrm{pk}}\right|$ and RC. The only discrepancy with this feature corresponds to the BNanoperm series, for which $\left|\Delta S_{M}^{\mathrm{pk}}\right|$ remains practically constant for the studied samples, making it a candidate for constituent of composite refrigerants. This constant value of $\left|\Delta S_{M}^{\mathrm{pk}}\right|$ has been recently ascribed to the increasing effective magnetic moment of $\mathrm{Fe}$ with increasing B content in the alloy. ${ }^{17}$

It is seen that the optimal alloy series of those presented here is the CoNanoperm, as it maximizes the refrigerant capacity maintaining $\left|\Delta S_{M}^{\mathrm{pk}}\right|$ in the upper part of the scale. A simple estimate of the adiabatic temperature change by means of the temperature dependence of the zero field specific heat of the $\mathrm{Fe}_{78} \mathrm{Co}_{5} \mathrm{Zr}_{6} \mathrm{~B}_{10} \mathrm{Cu}_{1}$ sample (the one with the maximum $\mathrm{RC}$ in the present study) yields a maximum value of $1.3 \mathrm{~K}$ for an applied field of $1.5 \mathrm{~T}$ (which, for the sake of comparison with other high temperature magnetic refrigerant materials, extrapolates to $3.2 \mathrm{~K}$ for a field of $5 \mathrm{~T}$ ).

Despite the diversity in the shape of the $\Delta S_{M}$ curves for the studied alloys, all of them share the same field dependence, expressed as $\Delta S_{M} \propto H^{n}$. As an example, Fig. 2 shows the temperature dependence of $n$ for two CoBAA alloys, showing a quadratic field dependence $(n=2)$ in the paramagnetic regime, a linear dependence $(n=1)$ in the ferromagnetic

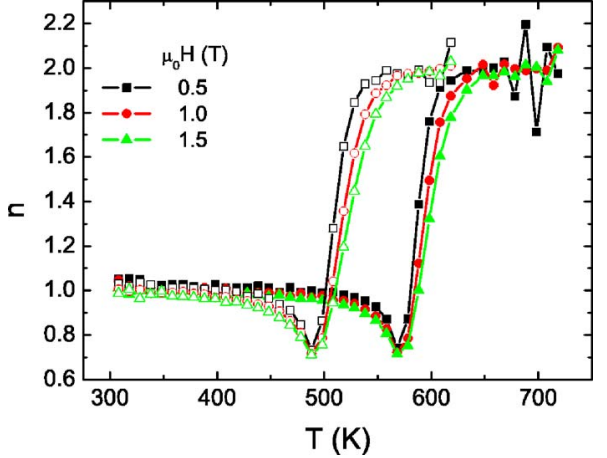

FIG. 2. (Color online) Temperature dependence of the exponent controlling the field dependence of the magnetic entropy change for $\mathrm{Fe}_{29} \mathrm{Co}_{40} \mathrm{~B}_{9} \mathrm{C}_{2} \mathrm{Si}_{3} \mathrm{Al}_{5} \mathrm{Ga}_{2} \mathrm{P}_{10}$ (open symbols) and $\mathrm{Fe}_{56} \mathrm{Co}_{14} \mathrm{~B}_{6} \mathrm{C}_{4} \mathrm{Si}_{3} \mathrm{Al}_{5} \mathrm{Ga}_{2} \mathrm{P}_{10}$ (solid symbols) for three values of the maximum applied field.

temperature range, and a potential law with a field exponent $n \sim 0.71$ at the Curie temperature. These values of $n$ have been ascribed ${ }^{16}$ to the Curie-Weiss law at high $T$, to the temperature independence of the field dependence of magnetization at low $T$, and to $n=1+(1 / \delta)[1-(1 / \beta)]$ at $T_{C}$, where $\beta$ and $\delta$ are the critical exponents. Taking into account that the critical exponents remain practically constant for alloys of similar compositional series, it is expected that the recently proposed master curve for the field dependence of $\Delta S_{M}$ (Ref. 16) also holds for different alloys of these series. As an example, Fig. 3 shows the normalized magnetic entropy change of the alloys for which $1 / 2 \Delta S_{M}^{\mathrm{pk}}$ is inside the experimental temperature range (CoBAA except $x=5$; CrMoBAA; CoNanoperm; $x=20$ BNanoperm; MnHiTperm except $x=0$, and MoFinemet) as a function of the rescaled temperature $\theta$, defined as

$$
\theta= \begin{cases}-\left(T-T_{C}\right) /\left(T_{r 1}-T_{C}\right), & T \leqslant T_{C} \\ \left(T-T_{C}\right) /\left(T_{r 2}-T_{C}\right), & T>T_{C},\end{cases}
$$

where $T_{r 1}$ and $T_{r 2}$ are the temperatures of the two reference points of each curve that correspond to $1 / 2 \Delta S_{M}^{\mathrm{pk}}$. The inset shows the original data of the CoBAA series used to construct this master curve. Further work to study the extension

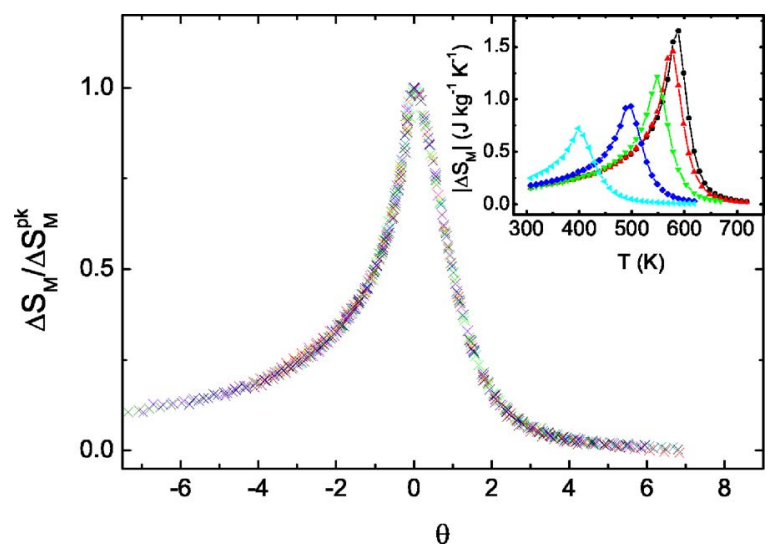

FIG. 3. (Color online) Inset: Temperature dependence of the magnetic entropy change of the CoBAA series with $x=70,56,43,29$, and 17, for a maximum applied field of $1.5 \mathrm{~T}$. Main panel: Master curve behavior of all the alloys for which $\frac{1}{2} \Delta S_{M}^{\mathrm{pk}}$ is inside the experimental temperature range (16 curves). 
of this master curve to different systems with larger differences in the critical exponents is being undertaken.

In conclusion, the magnetocaloric response of alloy ribbons from Finemet, Nanoperm, HiTperm, and bulk amorphous families has been presented. The optimal composition of those studied in this paper corresponds to a Co-containing Nanoperm alloy, for which the maximum magnetic entropy change is $1.6 \mathrm{~J} /(\mathrm{kg} \mathrm{K})$ for an applied field of $1.5 \mathrm{~T}$, with a corresponding refrigerant capacity of $82 \mathrm{~J} / \mathrm{kg}$ and an estimated adiabatic temperature change of $1.3 \mathrm{~K}$. These values extrapolate to $3.9 \mathrm{~J} /(\mathrm{kg} \mathrm{K}), 273 \mathrm{~J} / \mathrm{kg}$, and $3.2 \mathrm{~K}$ for an applied field of $5 \mathrm{~T}$, respectively. It has also been shown that the field dependence of $\Delta S_{M}$ follows a general trend for soft magnetic amorphous alloys, giving rise to a master curve behavior for alloys of similar compositional series.

This work was supported by the Spanish Government and EU-FEDER (Project MAT 2004-04618) and the PAI of Junta de Andalucía. One of the authors (J.S.B.) is grateful to Junta de Andalucía for a research contract.

${ }^{1}$ A. M. Tishin, in Handbook of Magnetic Materials, edited by K. H. J. Buschow (Elsevier, Amsterdam, 1999), Vol. 12, pp. 395-524.

${ }^{2}$ K. A. Gschneidner, Jr. and V. K. Pecharsky, Annu. Rev. Mater. Sci. 30, 387 (2000)

${ }^{3}$ E. Brück, J. Phys. D 38, R381 (2005).
${ }^{4}$ C. A. Zimm, A. Jastrab, A. Sternberg, V. K. Pecharsky, K. A. Gschneidner, Jr., M. G. Osborne, and I. E. Anderson, Adv. Cryog. Eng. 43, 1759 (1998).

${ }^{5}$ D. Wang, K. Peng, B. Gu, Z. Han, S. Tang, W. Qin, and Y. Du, J. Alloys Compd. 358, 312 (2003).

${ }^{6}$ S. Atalay, H. Gencer, and V. S. Kolat, J. Non-Cryst. Solids 351, 2373 (2005).

${ }^{7}$ S. G. Min, K. S. Kim, S. C. Yu, H. S. Suh, and S. W. Lee, J. Appl. Phys. 97, 10M310 (2005).

${ }^{8}$ V. Franco, J. S. Blázquez, C. F. Conde, and A. Conde, Appl. Phys. Lett. 88, 042505 (2006).

${ }^{9}$ F. Johnson and R. D. Shull, J. Appl. Phys. 99, 08K909 (2006).

${ }^{10}$ V. Franco, J. S. Blázquez, and A. Conde, J. Appl. Phys. 100, 064307 (2006).

${ }^{11}$ V. Franco, J. M. Borrego, A. Conde, and S. Roth, Appl. Phys. Lett. 88, 132509 (2006).

${ }^{12}$ V. Franco, J. M. Borrego, C. F. Conde, A. Conde, M. Stoica, and S. Roth, J. Appl. Phys. 100, 083903 (2006).

${ }^{13}$ Q. Luo, D. Q. Zhao, M. X. Pan, and W. H. Wang, Appl. Phys. Lett. 89, 081914 (2006).

${ }^{14}$ V. K. Pecharsky and K. A. Gschneidner, Jr., Phys. Rev. Lett. 78, 4494 (1997).

${ }^{15}$ V. Provenzano, A. J. Shapiro, and R. D. Shull, Nature (London) 429, 853 (2004).

${ }^{16}$ V. Franco, J. S. Blázquez, and A. Conde, Appl. Phys. Lett. 89, 222512 (2006).

${ }^{17}$ V. Franco, C. F. Conde, J. S. Blázquez, A. Conde, P. Švec, and D. Janičkovič (to be published).

${ }^{18}$ V. Franco, C. F. Conde, J. S. Blázquez, M. Millán, and A. Conde (unpublished).

${ }^{19}$ M. E. Wood and W. H. Potter, Cryogenics 25, 667 (1985). 